\title{
Comunicación
}

\section{VALORES HEMATOLÓGICOS DEL PAICHE Arapaima gigas DE LA AMAZONÍA PERUANA}

\author{
Haematological Values of Paiche Arapaima gigas in the Peruvian Amazonia \\ Enrique Serrano M. ${ }^{1,2}$, Guillermo Leguía P. ${ }^{1}$, Marco Quispe H. ${ }^{1}$, Gina Casas V. ${ }^{1}$
}

\section{Resumen}

Se determinaron los valores hematológicos de paiche, Arapaima gigas, en 120 muestras de sangre colectadas entre mayo de 2009 y enero de 2011. Los peces procedían de dos centros de cultivo piscícola de la provincia de Maynas, Loreto, Perú. Las muestras fueron clasificadas de acuerdo al grupo etario: 10-30, 31-180, 181-365 y > 365 días. Se obtuvieron valores de recuento de glóbulos rojos y blancos, hematocrito y hemoglobina, así como el recuento diferencial de leucocitos. Los valores de glóbulos rojos y de hemoglobina se incrementaron con la edad $(\mathrm{p}<0.05)$.

Palabras clave: Arapaima, hematología, Amazonía peruana, paiche, acuicultura

\section{Abstract}

Haematological values of paiche, Arapaima gigas, were determined in 120 blood samples collected from May 2009 to January 2011. The fishes were cultivated in two fish farms located in the province of Maynas, Loreto, Peru. Blood samples were classified according to four age groups: 10-30, 31-180, 181-365 and > 365 days. Data for erythrocyte and leukocyte counts, haemoglobin, haematocrit and differential leukocyte count were obtained. Red cell count and haemoglobin increased with age $(\mathrm{p}<0.05)$.

Key words: Arapaima, haematology, Peruvian Amazonia, paiche, aquaculture

\footnotetext{
${ }^{1}$ Grupo SALUVET-UPCH, Facultad de Veterinaria y Zootecnia, Universidad Peruana Cayetano Heredia, Lima

${ }^{2}$ E-mail: enrique.serrano@upch.pe
} 


\section{INTRODUCCIÓN}

El paiche Arapaima gigas es uno de los dos peces más grandes de América del Sur, pudiendo llegar a medir más de $2 \mathrm{~m}$ de longitud y pesar más de $100 \mathrm{~kg}$. Es una especie de importancia en la economía pesquera del Amazonas.

En el Perú, el paiche es fuente de alimentación y generador de ingresos para el poblador amazónico a través de la comercialización de sus productos. La pesca sin control ha mermado su presencia en los ríos, de modo que las autoridades locales y regionales han iniciado una exhaustiva promoción del cultivo de esta especie en ambientes controlados; sin embargo, la crianza en piscigranjas predispone a situaciones de estrés al animal, repercutiendo en su normal desarrollo.

El conocimiento sobre los valores hematológicos de paiches cultivados en la Amazonía peruana es escaso; sin embargo, estudios realizados en Brasil señalan una asociación entre los parámetros hematológicos y el estadio de desarrollo del pez (TavaresDias y Moraes, 2004; Tavares-Dias et al., 2008). Los valores hematológicos de esta especie son una herramienta útil para determinar su condición sanitaria (Tavares-Dias y Moraes, 2004, 2007; Tavares-Dias et al., 2007, 2008; Davis et al. 2008). Por ello, el objetivo del presente estudio fue determinar los valores hematológicos en paiches en varios estadios de desarrollo provenientes de centros de cultivo localizados en la Amazonía Peruana.

\section{Materiales y Métodos}

Entre mayo de 2009 y enero de 2011 se colectaron muestras de sangre de 120 paiches criados en cautiverio en dos centros de cultivo localizados en la carretera Iquitos-Nauta, en la provincia de Maynas, Loreto, Perú.
Los peces se encontraban en aparente buen estado de salud. Las muestras se colectaron por punción de la vena caudal mediante el empleo de jeringas de 1 y $3 \mathrm{ml}$ conteniendo EDTA $10 \%$ en el caso de alevinos y juveniles, respectivamente, y de microcapilares con EDTA para los peces más pequeños.

Se hizo el contaje de eritrocitos en la cámara de Neubauer, la determinación de hematocrito por el método de microhematocrito y la concentración de hemoglobina por el método de oxihemoglobina. Además, se hicieron frotices sanguíneos para determinar el número de leucocitos totales y para el contaje diferencial de leucocitos. El procesamiento de las muestras se llevó a cabo en el Laboratorio de Patología Clínica de la Facultad de Veterinaria y Zootecnia de la Universidad Peruana Cayetano Heredia, Lima.

Las muestras fueron clasificadas según el grupo etario: 10-30, 31-180, 181-365, >365 días de edad. La talla de los peces varió desde los 7 a $104 \mathrm{~cm}$ y el peso de 7.7 a $2246 \mathrm{~g}$. En la comparación de las variables con los estadios de desarrollo se empleó la prueba «t» de Student, asumiéndose un intervalo de confianza de $95 \%(\mathrm{p}<0.05)$.

\section{Resultados y Discusión}

Los resultados muestran que los valores de glóbulos rojos y hemoglobina se incrementan con la edad (Cuadro 1), lo cual concuerda con hallazgos en esturiones $A$. percicus y H. huso (Bahmani et al., 2001); sin embargo, Drumond et al. (2010) trabajando con paiche y Centeno et al. (2007) con Colossoma macropomum observaron mayor concentración de glóbulos rojos y hematocrito en alevinos en comparación con juveniles. El incremento en el hematocrito y concentración de glóbulos rojos es esperado, toda vez que hay un crecimiento de los tejidos hematopoyéticos (riñón y bazo) en los peces jóvenes (Hrubec et al., 2001). 
Cuadro 1. Valores hematológicos (promedio \pm intervalo de confianza) en paiche Arapaima gigas, según el grupo etario (30 especímenes por grupo)

\begin{tabular}{lcccc}
\hline & \multicolumn{4}{c}{ Edad en días } \\
\cline { 2 - 5 } & $10-30$ & $31-180$ & $181-365$ & $>365$ \\
\hline Eritrocitos (x10 $\left.{ }^{6}\right)$ & $1.42 \pm 0.21^{\mathrm{a}}$ & $1.33 \pm 0.02^{\mathrm{a}}$ & $1.92 \pm 0.16^{\mathrm{a}}$ & $2.62 \pm 0.42^{\mathrm{a}}$ \\
Hematocrito (\%) & $32.1 \pm 1.8^{\mathrm{a}}$ & $25.2 \pm 0.5^{\mathrm{b}}$ & $34.8 \pm 1.1^{\mathrm{a}}$ & $30.8 \pm 1.5^{\mathrm{a}}$ \\
Hemoglobina (g/dl) & $8.67 \pm 0.45^{\mathrm{a}}$ & $7.74 \pm 0.21^{\mathrm{a}}$ & $8.42 \pm 0.61^{\mathrm{a}}$ & $9.10 \pm 0.32^{\mathrm{a}}$ \\
Leucocitos (x10) & $12.27 \pm 1.26^{\mathrm{a}}$ & $32.39 \pm 0.33^{\mathrm{b}}$ & $15.46 \pm 1.85^{\mathrm{a}}$ & $15.84 \pm 5.48^{\mathrm{a}}$ \\
Diferencial & & & & \\
$\quad$ Abastonados (\%) & $0.2 \pm 0.2$ & $0.8 \pm 0.1$ & $0.1 \pm 0.1$ & $0.4 \pm 0.2$ \\
$\quad$ Segmentados (\%) & $73.7 \pm 2.4$ & $84.5 \pm 0.9$ & $77.7 \pm 2.0$ & $68.9 \pm 4.5$ \\
$\quad$ Linfocitos (\%) & $17.5 \pm 2.6$ & $83.8 \pm 3.5$ & $16.2 \pm 2.0$ & $35.7 \pm 7.5$ \\
Monocitos (\%) & $0.2 \pm 0.1$ & $4.2 \pm 0.4$ & $0.1 \pm 0.1$ & $0.6 \pm 0.3$ \\
Eosinófilos (\%) & $3.4 \pm 0.8$ & $7.1 \pm 0.4$ & $5.6 \pm 1.4$ & $2.7 \pm 2.2$ \\
Basófilos (\%) & $0.4 \pm 0.2$ & $2.1 \pm 0.3$ & $0.6 \pm 0.4$ & $1.0 \pm 0.4$ \\
\hline
\end{tabular}

a,b Superíndices diferentes dentro de parámetros hematológicos indican diferencia estadística $(p<0.05)$

Los linfocitos, monocitos, neutrófilos y eosinófilos en los frotis sanguíneos tuvieron características morfológicas similares a las descritas por otros investigadores en juveniles de paiche (Tavares-Dias et al., 2007; Drumond et al., 2010). Asimismo, se observó la mayor concentración de leucocitos en paiches entre 31 a 180 días de edad $(\mathrm{p}<0.05)$.

\section{Agradecimiento}

El presente trabajo fue desarrollado mediante el apoyo financiero de la Presidencia del Consejo de Ministros a través del FINCyT (Contrato N. ${ }^{\circ}$ 017-FINCyTPIBAP-2009). Asimismo, se agradece a los paichecultores Sr. Wenceslao Solsol y Sr. Manuel García por su valioso apoyo en el estudio.

\section{Literatura Citada}

1. Bahmani M, Kazemi R, Donskaya P. 2001. A comparative study of some hematological features in young reared sturgeons (Acipenser persicus and Huso huso). Fish Physiol Biochem 24: 135-140.

2. Centeno L, Silva-Acuña $R$, Barrios $R$, Lugo RS, Matute C, Pérez JL. 2007. Hematological characteristics of cachama (Colossoma macropomum) in three phases of the growth in Delta Amacuro, Venezuela. Zootec Trop 25: 237-243.

3. Davis AK, Maney DL, Maerz JC. 2008. The use of leukocytes profiles to measure stress in vertebrates: a review for ecologists. Funct Ecol 22: 760-772. 
4. Drumond GVF, Caixeiro AP, TavaresDias M, Marcon JL, Affonso EG. 2010. Características bioquímicas e hematológicas do pirarucu Arapaima gigas Schinz, 1822 (Arapaimidae) de cultivo semi-intensivo na Amazônia. Acta Amazônica 40: 591-596.

5. Hrubec TC, Smith SA, Robertson JL. 2001. Age-related changes in hematology and plasma chemistry values of hybrid striped bass (Morone chrysops $\mathrm{x}$ Morone saxatatilis). Vet Clin Pathol 30: 8-15.

6. Tavares-Dias M, Affonso EG, Oliveira SR, Marcon JL, Egami MI. 2008. Comparative study on hematological parameters of farmed matrinxã, Brycon amazonicus Spix and Agassiz, 1829
(Characidae: Bryconinae) with others Bryconinae species. Acta Amazônica 38: 799-806.

7. Tavares-Dias M, Barcellos JFM, Marcon JL, Menezes GC, Ono EA, Affonso EG 2007. Hematological and biochemical parameters for the pirarucu Arapaima gigas Schinz, 1822 (Osteoglossiformes, Arapaimidae) in net cage culture. Electronic J Ichthyol 2: 61-68.

8. Tavares-Dias M, Moraes FR. 2004. Hematology of teleosts fish. Ribeirão Preto: Villimpress. 144 p.

9. Tavares-Dias M, Moraes FR. 2007. Haematological and biochemical reference intervals for farmed channel catfish. J Fish Biol 71: 383-388. 